\title{
Optimal Utility Lifetime and Delay-Robustness Tradeoff in Wireless Multimedia Sensor Networks
}

\author{
Muhammad Tahir* and Ronan Farrell ${ }^{\dagger}$ \\ *Dept. of Electrical Eng. Univ. of Engineering and Technology Lahore \\ $\dagger$ Institute of Microelectronics and Wireless Systems \\ National University of Ireland Maynooth, Ireland \\ mtahir@uet.edu.pk, rfarrell@eeng.nuim.ie
}

\begin{abstract}
A unified framework to achieve optimal network lifetime, utility and delay-robustness tradeoff, for wireless multimedia sensor networks, is proposed. Using delay-robustness gives the flexibility to achieve any desired level of delay QoS provisioning. We have employed sensitivity analysis to define an appropriate objective function for delay-robustness. This is achieved by capturing delay-robustness in the end-to-end delay constraints and penalizing its price in the objective function. For distributed realization of the proposed framework, the optimal tradeoff problem is decomposed into lifetime, utility and delayrobustness subproblems. Performance evaluation results show that compromising network utility can not provide both lifetime and delay-robustness, simultaneously, for all operating points.
\end{abstract}

\section{INTRODUCTION}

Due to the availability of low cost imaging sensors, wireless multimedia sensor networks (WMSN) can now potentially enable a large class of applications ranging from image-based tracking and surveillance to smart environments and elderly assistance in public spaces [1]. There is an inherent need for maximizing the network lifetime when the nodes in these networks are battery powered. At the same time performance demands in WMSNs try to maximize network utility to improve the quality-of-service $(\mathrm{QoS})$. This points towards the network utility-lifetime tradeoff [2]. In [3] joint network utility and lifetime maximization problem is formulated as a cross-layer optimization problem and a distributed solution is proposed using dual decomposition. The authors in [2] investigate the interaction between energy consumption and rate allocation as a constrained optimization problem, which provides lifetime maximization and network utility tradeoff. Optimal flow control to achieve utility-lifetime tradeoff is discussed in [4], where the authors take into account link congestion and energy efficiency and introduce inconsistent coordination price to balance the energy consumption among sensor nodes. The above mentioned utility-lifetime tradeoff problems do not consider the effect of this tradeoff on the delay incurred in transporting the data, which is also of prime importance in WMSNs. In fact, one of the key objectives in WMSNs is the end-to-end delay QoS provisioning.

The problem of lifetime-delay tradeoff has been considered separately in literature. Authors in [5] study the tradeoff between network lifetime and end-to-end delay, by employing an objective of minimizing energy dissipation when subjected to delay constraints. A weighted fair queuing based mechanism for data aggregation, subject to end-to-end delay constraints, is proposed in [6], which achieves service differentiation and also ensures delay bounds. The techniques proposed in [5] and [6] can achieve an optimal lifetime-delay tradeoff, but fall short in achieving an optimal utilization of the network resources. In addition, improving the delay QoS performance by minimizing the end-to-end delays arbitrarily, can compromise the network utility significantly. On the other hand maximizing network utilization can degrade both network lifetime as well as delay QoS performance. This intrinsic tradeoff between network utility, lifetime and delay performance can be achieved optimally using the proposed unified framework.

Traditionally end-to-end delay performance is obtained by requiring maximum delay thresholds to be met. But doing so can lead to a solution, where end-to-end delays approach maximum delay thresholds. This approach provides a solution where optimal performance is achieved at the expense of a vulnerable WMSN, which is prone to performance degradation due to delay threshold violations. To address this issue, our approach is based on delay-robustness, which provides delay margin, a measure of the gap between optimal end-toend delay and corresponding delay threshold, by introducing parameter $\omega$ in the end-to-end delay constraints. Using delayrobustness, rather than meeting delay thresholds gives flexibility to achieve any desired level of delay QoS provisioning. However the introduction of parameter $\omega$ is at the expense of slightly degraded utility and lifetime performance. To realize the lifetime, utility and delay-robustness tradeoff, we have formulated a multi-objective optimization problem, which captures delay-robustness in the end-to-end delay constraints through parameter $\omega$ and its price is penalized in the objective function. For a given network utility objective function, we have employed sensitivity analysis to determine the optimal objective function for delay-robustness. The proposed solution provides the application a unified framework to achieve an optimal tradeoff between contending network utility, lifetime and delay-robustness objectives.

\section{SySTEM MODEL}

The network is modeled as a tree graph $G(N, L)$, where $N$ and $L$ are, respectively, sets of sensor nodes (excluding the sink node $n_{\text {sink }}$ ) and communication links. Each sensor node $n_{i}, n_{i} \in N$ has an uncompressed data arrival rate, $R_{n_{i}}$, from 
its sensors. The node compresses the data before transmission to exploit the sensor data correlation and the resulting compressed data rate is $r_{n_{i}}^{(i n)} \in \mathbf{r}^{(i n)}$. The compressed data from the local sensor as well as from the sensors in the node's subtree can be transmitted at a transmission rate of $r_{n_{i}}^{(\text {out })} \in \mathbf{r}^{(\text {out })}$. We use a variable data transmission rate, as most of the wireless transceivers today provide a range of data transmission rates ${ }^{1}$. The set $I\left(n_{i}\right)$ represents the collection of nodes in the subtree of node $n_{i}$, while $T\left(n_{i}\right)$ is the set of nodes along the shortest path from $n_{i}$ to the $n_{\text {sink }}$.

To evaluate network lifetime we need to find maximum power consumption at a sensor node. To do that, power consumption at each sensor node, $n_{i}$, is obtained from communication power $\left(P_{n_{i}}^{(c o m m)}\right)$ and computation power $\left(P_{n_{i}}^{(c o m p)}\right)$ costs. The communication power consumption, for a tree like network topology, is given by

$$
P_{n_{i}}^{(\text {comm })}\left(\mathbf{r}^{(\text {out })}\right)=E_{b}^{(t x)} r_{n_{i}}^{(\text {out })}+E_{b}^{(r x)} \sum_{n_{j} \in I\left(n_{i}\right)} r_{n_{j}}^{(\text {out })} .
$$

In (1) $E_{b}^{(t x)}$ and $E_{b}^{(r x)}$ are, respectively, per bit transmission and reception energy costs. The computational power consumption is proportional to compression ratio $R_{n_{i}} / r_{n_{i}}^{(i n)}$ and uncompressed data rate $R_{n_{i}}$, [7] and is given by [8]

$$
P_{n_{i}}^{(c o m p)}\left(\mathbf{r}^{(i n)}\right)=R_{n_{i}}\left[\left(R_{n_{i}} / r_{n_{i}}^{(i n)}\right)^{\alpha}-1\right] E_{b}^{(c o m p)}+\epsilon .
$$

In (2), $E_{b}^{(c o m p)}$ is the per bit computational energy cost, $\alpha>0$ depends on the choice of compression algorithm and $\epsilon$ is the offset for boundary value compensation. For a given compression algorithm and the hardware platform, parameters $\alpha$ and $\epsilon$ are obtained off-line. The computational power consumption model is validated in [8] for the variable wavelet transform level compression proposed in [9] and is also used to obtain $\alpha$ and $\gamma$ parameters. Combining the computation and communication power costs, the total power consumption for node $n_{i}, P_{n_{i}}$, is given by

$$
P_{n_{i}}\left(\mathbf{r}^{(\text {out })}, \mathbf{r}^{(i n)}\right)=P_{n_{i}}^{(\text {comm })}\left(\mathbf{r}^{(\text {out })}\right)+P_{n_{i}}^{(\text {comp })}\left(\mathbf{r}^{(i n)}\right) .
$$

To provide delay quality of service (QoS) guarantee, mean end-to-end delay along the path $T\left(n_{i}\right)$ is upper bounded by the threshold $D_{\max }\left(n_{i}\right)$. The end-to-end delay, for packet length $H$, is obtained from each link delay along the path $T\left(n_{i}\right)$ using $M / M / 1$ queuing model [10] and is given by

$$
\sum_{n_{k} \in T\left(n_{i}\right)} \frac{H}{r_{n_{k}}^{(o u t)}-\left(r_{n_{k}}^{(\text {in })}+\sum_{n_{j} \in I\left(n_{k}\right)} r_{n_{j}}^{(\text {out })}\right)} \leq D_{\max }\left(n_{i}\right) .
$$

\section{Optimal LifETIME UTILITY AND DELAY-ROBUSTNESS TRADEOFF}

To formulate optimal lifetime, utility and delay-robustness tradeoff problem we decompose the end-to-end delay constraint in (4), using auxiliary variables $d_{n_{k}} \in \mathbf{d}$, into multiple

\footnotetext{
${ }^{1}$ For instance, Texas Instruments $\mathrm{CC} 1101$ has data transmission rates from $1.2 \mathrm{kbps}$ to $500 \mathrm{kbps}$. Same is true for IEEE 802.11 data transceivers.
}

link delays along $T\left(n_{i}\right)$ as

$$
\begin{array}{r}
\sum_{n_{k} \in T\left(n_{i}\right)} d_{n_{k}} \leq D_{\max }\left(n_{i}\right), \\
\frac{H}{d_{n_{k}}} \leq r_{n_{k}}^{(\text {out })}-\left(r_{n_{k}}^{(\text {in })}+\sum_{n_{j} \in I\left(n_{k}\right)} r_{n_{j}}^{\text {(out })}\right) .
\end{array}
$$

Decomposing the end-to-end delay into multiple link delays enables us to achieve a distributed implementation of optimal lifetime, utility and delay-robustness tradeoff problem. Now we introduce delay-robustness parameter, $\omega_{n_{i}} \in \boldsymbol{\Omega}: \omega_{n_{i}} \in$ $[0,1) \forall n_{i}$, to achieve optimal tradeoff by modifying (5) as

$$
\sum_{n_{k} \in T\left(n_{i}\right)} d_{n_{k}} \leq\left(1-\omega_{n_{i}}\right) D_{\max }\left(n_{i}\right),
$$

The parameter $\omega_{n_{i}}$ is upper bounded by 1 due to the fact that the data transmission rate $r_{n_{i}}^{(\text {out })}$ can not be increased arbitrarily and the lower bound ensures that the delay threshold $D_{\max }\left(n_{i}\right)$ is not satisfied impractically by using negative values of $\omega_{n_{i}}$. Now the lifetime, utility and delay-robustness tradeoff problem is formulated as

$\operatorname{maximize}\left\{\min \frac{\beta}{P_{n_{i}}}+\sum_{n_{i}}\left(\beta \theta\left(\omega_{n_{i}}\right)+(1-\beta) U\left(r_{n_{i}}^{(i n)}\right)\right)\right\}$

$$
\begin{aligned}
& \text { s.t. } \sum_{n_{k} \in T\left(n_{i}\right)} d_{n_{k}} \leq\left(1-\omega_{n_{i}}\right) D_{\max }\left(n_{i}\right) \forall n_{i} \\
& \frac{H}{d_{n_{i}}} \leq r_{n_{i}}^{(\text {out })}-\left(r_{n_{i}}^{(\text {in })}+\sum_{n_{j} \in I\left(n_{i}\right)} r_{n_{j}}^{(\text {out })}\right) \forall n_{i} \\
& 0 \leq \omega_{n_{i}}<1, R_{n_{i}} / r_{n_{i}}^{(\text {in })} \leq \gamma_{\max }, r_{n_{i}}^{(\text {out })} \leq R_{\max } \forall n_{i} .
\end{aligned}
$$

In (8), $\theta($.$) and U($.$) are, respectively, delay-robustness and$ utility objective functions, $R_{\max }$ is maximum data transmission rate capability of RF interface and $\beta$ is weighting coefficient to achieve the desired level of tradeoff. We also impose an upper bound, $\gamma_{\max }$, on compression ratio, $R_{n_{i}} / r_{n_{i}}^{(i n)}$, to limit data distortion due to lossy compression. An equivalent formulation, which is suitable for distributed realization, is obtained through problem transformation to an equivalent epigraph form by introducing the slack variable $t$ as follows,

$$
\begin{aligned}
\operatorname{maximize} & {\left[\beta t+\beta \sum_{n_{i}} \theta\left(\omega_{n_{i}}\right)+(1-\beta) \sum_{n_{i}} U\left(r_{n_{i}}^{(i n)}\right)\right] } \\
& P_{n_{i}} \leq 1 / t \forall n_{i}, \text { constraints }(9)-(11) \quad(12)
\end{aligned}
$$

The problem in (12) is decomposed for distributed realization by using dual variables $\lambda_{n_{i}} \in \boldsymbol{\Lambda}$ and $\psi_{n_{i}} \in \boldsymbol{\Psi}$, to obtain

$$
\begin{array}{r}
L\left(t, \mathbf{r}^{(\text {in })}, \mathbf{r}^{(\text {out })}, \mathbf{d}, \boldsymbol{\Omega}, \boldsymbol{\Lambda}, \boldsymbol{\Psi}\right)=\operatorname{maximize}\{\beta t+ \\
\sum_{n_{i}}\left(\beta \theta\left(\omega_{n_{i}}\right)+(1-\beta) U\left(r_{n_{i}}^{(i n)}\right)+\lambda_{n_{i}}\left(1 / t-P_{n_{i}}\right)\right) \\
\left.+\sum_{n_{i}} \psi_{n_{i}}\left(\left(1-\omega_{n_{i}}\right)-\frac{\sum_{n_{k} \in T\left(n_{i}\right)} d_{n_{k}}}{D_{\max }\left(n_{i}\right)}\right)\right\}
\end{array}
$$




$$
\text { s.t. constraints }(10)-(11) \text {. }
$$

The maximization problem in (13) is decomposable into delayrobustness, lifetime and joint utility and node power allocation subproblems. The associated dual problem is given by

$$
\begin{array}{cl}
\operatorname{minimize} & g(\boldsymbol{\Lambda}, \mathbf{\Psi}) \\
\text { s.t } & \lambda_{n_{i}}, \psi_{n_{i}} \geq 0 \quad \forall n_{i} .
\end{array}
$$

In (14) $g(\boldsymbol{\Lambda}, \boldsymbol{\Psi})=L\left(t^{*}, \mathbf{r}^{*(\text { in })}, \mathbf{r}^{*(o u t)}, \mathbf{d}^{*}, \boldsymbol{\Omega}^{*}, \boldsymbol{\Lambda}, \boldsymbol{\Psi}\right)$ and $t^{*}, \mathbf{r}^{*(\text { in })}, \mathbf{r}^{*(\text { out })}, \mathbf{d}^{*}$ and $\boldsymbol{\Omega}^{*}$ are optimal primal variables obtained by solving (13). Next we describe the solution approach for each of the subproblems.

\section{A. Delay-Robustness Sub-problem}

The delay-robustness sub-problem from (13) is given by

$$
\begin{array}{cl}
\operatorname{maximize} & \sum_{n_{i}}\left(\beta \theta\left(\omega_{n_{i}}\right)+\psi_{n_{i}}\left(1-\omega_{n_{i}}\right)\right) \\
\text { s.t. } & 0 \leq \omega_{n_{i}}<1 .
\end{array}
$$

For a given concave utility function $U\left(r_{n_{i}}^{(i n)}\right)$, the objective function $\theta\left(\omega_{n_{i}}\right)$ is responsible for modulating the delayrobustness tradeoff with network lifetime and utility. To achieve an optimal tradeoff, the objective function $\theta\left(\omega_{n_{i}}\right)$ should be chosen appropriately. As a first step towards this objective, we fix $\beta=0$ in (8) and use sensitivity analysis to study the effect of perturbing the end-to-end delay threshold $D_{\max }\left(n_{i}\right)$ on the optimal network utility.

Step 1 - Sensitivity Analysis: We perturb the end-to-end delay constraint for $n_{i}^{t h}$ node by $\xi_{n_{i}} \in \boldsymbol{\Xi}$ and observe its effect on optimal network utility defined as $\rho^{*}(\boldsymbol{\Xi})$ by solving the following perturbed optimization problem:

$$
\rho^{*}(\boldsymbol{\Xi})=\text { maximize } \sum_{n_{i}} U\left(r_{n_{i}}^{(i n)}\right)
$$

s.t. $\frac{\sum_{n_{k} \in T\left(n_{i}\right)} d_{n_{k}}}{D_{\max }\left(n_{i}\right)} \leq \xi_{n_{i}} \forall n_{i}$, and constraint in (12).

The $\xi_{n_{i}}=1 \forall n_{i}$, represents the unperturbed problem. The $0<\xi_{n_{i}} \leq 1$ and $\xi_{n_{i}}>1$, respectively, correspond to tightening and relaxing the delay constraint corresponding to $n_{i}^{t h}$ node. If $\psi_{n_{i}}^{*}$ corresponding to $\rho^{*}(\boldsymbol{\Xi}=\mathbf{1})$ represents the optimal value of the Lagrange multiplier associated with the unperturbed end-to-end delay constraint then fractional change in $\rho^{*}(\boldsymbol{\Xi}=\mathbf{1})$ due to $n_{i}^{\text {th }}$ constraint perturbation is obtained as

$$
\begin{aligned}
\frac{\rho^{*}(\boldsymbol{\Xi})-\rho^{*}(\mathbf{1})}{\rho^{*}(\mathbf{1})} & =\frac{\rho^{*}\left(\xi_{n_{i}} \mathbf{e}_{n_{i}}\right)-\rho^{*}(\mathbf{1})}{\rho^{*}(\mathbf{1})} \\
\frac{\Delta \rho^{*}(\boldsymbol{\Xi})}{\rho^{*}(\mathbf{1})} & =\left(\xi_{n_{i}}-1\right) \frac{\partial \rho^{*}(\mathbf{1}) / \partial \xi_{n_{i}}}{\rho^{*}(\mathbf{1})}+o\left(\xi_{n_{i}}\right) \\
& =\left(\xi_{n_{i}}-1\right)\left(\psi_{n_{i}}^{*} / \rho^{*}(\mathbf{1})\right)+o\left(\xi_{n_{i}}\right) \\
& \approx\left(\xi_{n_{i}}-1\right) \frac{\psi_{n_{i}}^{*}}{\rho^{*}(\mathbf{1})}
\end{aligned}
$$

The vector $\mathbf{e}_{n_{i}}$ in the first equality of (17) has its $n_{i}^{t h}$ entry equal to one while all other being zero. The second equality of (17) follows from the Taylor series expansion, where $o\left(\xi_{n_{i}}\right)$ represents the second and higher order terms lumped together. The third equality of (17) is based on the fact that $\partial \rho^{*}(\mathbf{1}) / \partial u_{s_{i}}=\psi_{s_{i}}^{*}$ (see [11] Sec. 5.6), which is followed by first order approximation in the last expression of (17).

Step 2 - Choosing Delay-Robustness Objective Function: For optimal choice of the delay-robustness objective function, we differentiate the objective in (15) with respect to $\omega_{n_{i}}$ and set it equal to zero to obtain

$$
\left.\frac{\partial \theta\left(\omega_{n_{i}}\right)}{\partial \omega_{n_{i}}}\right|_{\omega_{n_{i}}=\omega_{n_{i}}^{*}}=\frac{\psi_{n_{i}}^{*}}{\beta} .
$$

From (18) we have $\psi_{n_{i}}^{*}=\beta \partial \theta\left(\omega_{n_{i}}\right) /\left.\partial \omega_{n_{i}}\right|_{\omega_{n_{i}}=\omega_{n_{i}}^{*}}$. Now in (17), substituting $\xi_{n_{i}}$ with $\left(1-\omega_{n_{i}}\right)$ and using $\psi_{n_{i}}^{*}=$ $\beta \partial \theta\left(\omega_{n_{i}}\right) /\left.\partial \omega_{n_{i}}\right|_{\omega_{n_{i}}=\omega_{n_{i}}^{*}}$, we have

$$
\begin{aligned}
\frac{\Delta \rho^{*}(\boldsymbol{\Xi})}{\rho^{*}(\mathbf{1})} & \approx\left(\xi_{n_{i}}-1\right) \frac{\psi_{n_{i}}^{*}}{\rho^{*}(\mathbf{1})} \\
& =-\left.\beta\left(\frac{\omega_{n_{i}}}{\rho^{*}(\mathbf{0})}\right) \frac{\partial \theta\left(\omega_{n_{i}}\right)}{\partial \omega_{n_{i}}}\right|_{\omega_{n_{i}}=\omega_{n_{i}}^{*}},
\end{aligned}
$$

where $\rho^{*}(\mathbf{0})$ is obtained by mapping $\rho^{*}(\mathbf{1})$ form $\xi_{n_{i}}$ to $\omega_{n_{i}}$ domain. By mapping $\xi_{n_{i}}$ to $\omega_{n_{i}}$ in the interval $(0,1]$, we ensure that $D_{\max }\left(n_{i}\right)$ is not violated. If $\delta_{n_{i}}=\left|\frac{\Delta \rho^{*}(\boldsymbol{\Xi})}{\rho^{*}(\mathbf{1})}\right|$ is the magnitude of the fraction of network utility objective, which is available for tradeoff with the end-to-end delay, then from (19) we have

$$
\delta_{n_{i}}=\omega_{n_{i}} \frac{\beta}{\rho^{*}(\mathbf{0})} \frac{\partial \theta\left(\omega_{n_{i}}\right)}{\partial \omega_{n_{i}}},
$$

and integrating (20) gives

$$
\theta\left(\omega_{n_{i}}\right)=\frac{\rho^{*}(\mathbf{0})}{\beta} \delta_{n_{i}} \log \left(\omega_{n_{i}}\right) .
$$

Using the result in (21), delay-robustness sub-problem becomes maximize $\sum_{n_{i}}\left(\rho^{*}(\mathbf{0}) \delta_{n_{i}} \log \left(\omega_{n_{i}}\right)+\psi_{n_{i}}(1-\right.$ $\left.\left.\omega_{n_{i}}\right)\right)$ s.t. $0 \leq \omega_{n_{i}}<1$. This problem can be solved in $\omega_{n_{i}}$ using efficient algorithms available for convex optimization [11].

\section{B. Lifetime Maximization Subproblem}

The network lifetime maximization subproblem from the Lagrangian function in (13) is given by

$$
\text { maximize } \beta t+\frac{\sum_{n_{i}} \lambda_{n_{i}}}{t} \text {. }
$$

Differentiating the objective in (22) with respect to $t$ and setting it to zero gives

$$
t^{*}=\sqrt{\frac{\sum_{n_{i}} \lambda_{n_{i}}}{\beta}}
$$

The condition $\lambda_{n_{i}} \geq 0$ in (14) can lead to small $t^{*}$, which in turn can result in arbitrary large $P_{n_{i}}$ due to $P_{n_{i}} \leq 1 / t$. But from a practical viewpoint, $P_{n_{i}}$ can not be increased arbitrarily high and an upper bound, $P_{n_{i}}^{(u b)}$, is imposed by the processor and the wireless transceiver used. This is ensured by introducing the constraint $P_{n_{i}}^{(u b)} \leq 1 / t^{*}$, which along with 
the result in (23) provides the solution for $t^{*}$ given by

$$
t^{*}=\min \left\{\frac{1}{P_{n_{i}}^{(u b)}}, \sqrt{\frac{\sum_{n_{i}} \lambda_{n_{i}}}{\beta}}\right\} .
$$

\section{Joint Utility and Power Allocation Subproblem}

From (13), the joint utility and node power allocation subproblem is given as

$$
\begin{array}{cl}
\operatorname{maximize} & \sum_{n_{i}}\left((1-\beta) U\left(r_{n_{i}}^{(i n)}\right)-\lambda_{n_{i}} P_{n_{i}}\right. \\
& \left.-\frac{\psi_{n_{i}}}{D_{\max }\left(n_{i}\right)} \sum_{n_{k} \in T\left(n_{i}\right)} d_{n_{k}}\right) \\
\text { s.t. } & \text { constraints }(10),(11) .
\end{array}
$$

Using the expression for $P_{n_{i}}$ from (3) and substituting for $P_{n_{i}}^{(c o m m)}$ and $P_{n_{i}}^{(c o m p)}$ the problem in (25) is convex in variables $r_{n_{i}}^{(\text {in })}, r_{n_{i}}^{(\text {out })}$ and $d_{n_{i}}$ and can be solved efficiently.

\section{Dual Problem}

The dual problem in (14) is solved by using the following projected sub-gradient updates:

$$
\begin{aligned}
\lambda_{n_{i}}(k+1) & =\left[\lambda_{n_{i}}(k)+\pi(k)\left(1 / t-P_{n_{i}}\right)\right]^{+} \forall n_{i},(26) \\
\psi_{n_{i}}(k+1) & =\left[\psi_{n_{i}}(k)+\pi(k)\left(\left(1-\omega_{n_{i}}\right)\right.\right. \\
& \left.\left.-\frac{\sum_{n_{k} \in T\left(n_{i}\right)} d_{n_{k}}}{D_{\max }\left(n_{i}\right)}\right)\right]^{+} \forall n_{i}
\end{aligned}
$$

In (26) and (27), $[x]^{+}$is defined as $\max \{0, x\}$ and $\left(t-1 / P_{n_{i}}\right)$ and $\left(\left(1-\omega_{n_{i}}\right)-D_{\max }^{-1}\left(n_{i}\right) \sum_{n_{k} \in T\left(n_{i}\right)} d_{n_{k}}\right)$ are the subgradients. We use variable step size rule $\pi(k)=1 / \sqrt{k}$, to update the dual variables [12]. The lifetime, utility and delayrobustness subproblems, coupled through dual variables, are assigned to different nodes, providing distributed realization.

\section{RESULTS}

To study the optimal tradeoff between network lifetime, utility and delay-robustness, we use the example network shown in Fig. 1. For the network utility, we have chosen a concave function, $U\left(r_{n_{i}}^{(i n)}\right)=\log \left(r_{n_{i}}^{(i n)}\right)$, which provides proportional throughput fairness among the nodes [3]. Due to a large number of possible combinations for different values of $R_{n_{i}}, \delta_{n_{i}}$ and $D_{\max }\left(n_{i}\right)$ for each sensor node, we use $R_{n_{i}}=R, \delta_{n_{i}}=\delta$ and $D_{\max }\left(n_{i}\right)=D_{\max }, \forall n_{i}$ without loss of generality. Different parameters used in the performance evaluation of the proposed optimal tradeoff framework are tabulated in Table I.

We first study the optimal network lifetime tradeoff with network utility and delay-robustness in Fig. 2 as a function of end-to-end delay threshold $D_{\max }$. As can be seen from the result in Fig. 2 that network lifetime increases when $D_{\max }$ increases. This is due to the fact that larger values of $D_{\max }$ allow a decrease in $r_{n_{i}}^{(o u t)}$ or an increase in $r_{n_{i}}^{(i n)}$ resulting in corresponding decrease in $P^{(\mathrm{comm})}$ or $P^{(\mathrm{comp})}$, respectively, which leads to network lifetime improvement. The variation

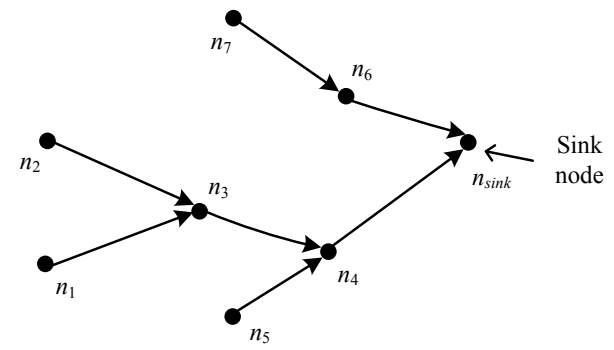

Fig. 1. An example WMSN consisting of seven sensor and single sink node.

TABLE I

Network PARAMETERS USED IN THE PERFORMANCE EVALUATION.

\begin{tabular}{c|c}
\hline \hline Parameters & Values \\
\hline \hline Transmission cost/bit $\left(E_{b}^{(t x)}\right)$ & $.209 \mu \mathrm{J} / \mathrm{bit}$ \\
\hline Reception cost/bit $\left(E_{b}^{(r x)}\right)$ & $.252 \mu \mathrm{J} / \mathrm{bit}$ \\
\hline Maximum transmission rate $\left(R_{\max }\right)$ & $500 \mathrm{kbps}$ \\
\hline Maximum compression ratio $\left(\gamma_{\max }\right)$ & 50 \\
\hline Packet length $(H)$ & $104 \mathrm{bytes}$ \\
\hline Computational cost/bit $\left(E_{b}^{(c o m p)}\right)$ & $.0023 \mu \mathrm{J} / \mathrm{bit}$ \\
\hline Uncompressed data rate $(R)$ & $500 \mathrm{kbps}$ \\
\hline Transmit power lower bound $\left(P_{n_{i}}^{(u b)}\right)$ & $10 \mathrm{~mW}$ \\
\hline Compression parameter $(\alpha)$ & 0.6 \\
\hline Compression offset parameter $(\epsilon)$ & 0.5 \\
\hline \hline
\end{tabular}

of parameter $\beta$ has an interesting affect on the network lifetime. One would expect increasing $\beta$ will lead to lifetime improvement, as it will assign more weight to the network lifetime (see (8)). This is true for $D_{\max }>55 \mathrm{~ms}$ as observed from the result in Fig. 2. But for smaller values of $D_{\max }$ increasing $\beta$ is counter productive resulting in degradation in the network lifetime. This is due to the fact that increasing $\beta$ also tries to improve the delay-robustness by increasing the delay margin. For $D_{\max }<55 \mathrm{~ms}$, the delay-robustness improvement, due to an increase in $\beta$, is achieved at the expanse of both network lifetime as well as network utility. On the other hand, for a $D_{\max }>55 \mathrm{~ms}$, both delay-robustness and lifetime improvements are achieved at the expanse of network utility. To verify this we use network throughput, defined as $\sum_{n_{i}} r_{n_{i}}^{(i n)}$, and is shown in Fig. 3 as a function of $D_{\max }$ and $\beta$. From the result in Fig. 3, we observe that increasing $\beta$ leads to throughput reduction, but a decrease of $0.45 \mathrm{Mbps}$ at $D_{\max }=15 \mathrm{~ms}$ only partially compensates for delayrobustness resulting in lifetime degradation. In contrast, a decrease of $0.786 \mathrm{Mbps}$ in the throughput at $D_{\max }=80 \mathrm{~ms}$ not only compensates for delay-robustness but also allows lifetime improvement. The lifetime improvement, due to an increase in $\beta$, for $D_{\max }>55 \mathrm{~ms}$ is based on the fact that for larger values of $D_{\max }$ higher compression ratio is achievable at the sensor nodes resulting in a decrease in $P^{(\mathrm{comm})}$ at the expense of an increase in $P^{(\mathrm{comp})}$. But a dominant decrease in $P^{(\mathrm{comm})}$ compared to a relatively small increase in $P^{(\mathrm{comp})}$ reduces node power $P_{n_{i}}$, leading to network lifetime improvement. This shows criticality of $D_{\max }$ in choosing $\beta$.

Finally the delay-robustness performance corresponding to 




Fig. 2. Network lifetime, $1 / t$, as a function of end-to-end delay threshold, $D_{\max }$. Normalization is done with the maximum value of $1 / t$ over its entire domain. Parameter $\delta=0.1$ is used.

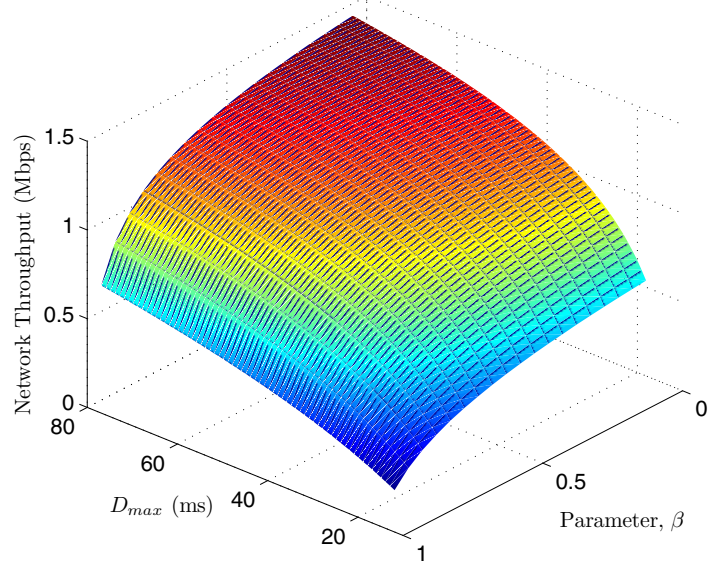

Fig. 3. Network throughput $\left(\sum_{n_{i}} r_{n_{i}}^{(i n)}\right)$ performance as a function of $D_{\max }$ and weighting coefficient $\beta$. Parameter $\delta=0.1$ is used for this result.

node $n_{4}$, is shown in Fig. 4 as a function of parameter $\delta$ for different values of $D_{\max }$. The result in Fig. 4 shows that significant delay-robustness improvement can be achieved by relaxing $D_{\max }$ when $D_{\max }$ is small, compared to marginal improvement for larger values of $D_{\max }$.

\section{CONCLUSIONS}

We have proposed a unified framework for wireless multimedia sensor networks to achieve optimal tradeoff among network lifetime, utility and delay-robustness. Sensitivity analysis is employed to define an appropriate objective function for delay-robustness providing any desired level of delay QoS provisioning. The multi-objective optimization problem is decomposed into lifetime, utility and delay-robustness subproblems providing distributed realization. Performance results provide an insight into the complex coupling among the contradicting objectives and show that how the network utility can be compromised to improve the lifetime and delay-robustness

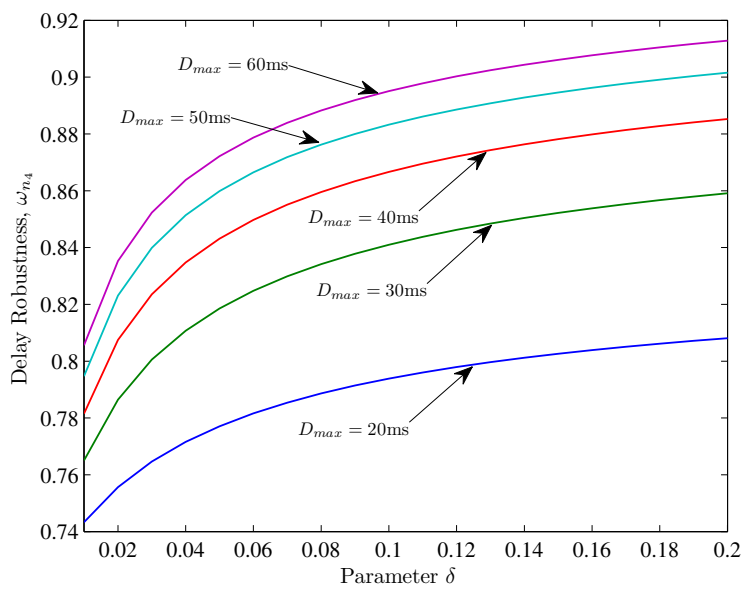

Fig. 4. Delay-robustness performance for node $n_{4}$ as a function of $\delta$ for different values of $D_{\max }$. Parameter $\delta$ chooses maximum utility fraction that we tradeoff with delay-robustness. We use $\beta=0.5$ for this result.

performance. The proposed framework can also be used for delay critical applications of sensor networks.

\section{ACKNOWLEDGMENTS}

Research presented in this paper was funded by a Strategic Research Cluster grant (07/SRC/I1168) by Science Foundation Ireland under the National Development Plan. The authors gratefully acknowledge this support.

\section{REFERENCES}

[1] I. Akyildiz, T. Melodia, and K. Chowdhury, "A survey on wireless multimedia sensor networks," Computer Networks, vol. 51, no. 4, pp. 921-960, 2007.

[2] J. Zhu, S. Chen, B. Bensaou, and K. Hung, "Tradeoff between lifetime and rate allocation in wireless sensor networks: A cross layer approach," in IEEE INFOCOM, 2007, pp. 267-275.

[3] H. Nama, M. Chiang, and N. Mandayam, "Utility-lifetime trade-off in self-regulating wireless sensor networks: a cross-layer design approach," in IEEE International Conference on Communications, 2006, pp. 35113516.

[4] J. Chen, S. He, Y. Sun, P. Thulasiraman, and X. Shen, "Optimal flow control for utility-lifetime tradeoff in wireless sensor networks," Computer Networks, to appear, 2009.

[5] Y. Yu, B. Krishnamachari, and V. Prasanna, "Energy-latency tradeoffs for data gathering in wireless sensor networks," in IEEE INFOCOM, 2004, pp. 244-255.

[6] K. Akkaya, M. Younis, and M. Youssef, "Efficient aggregation of delayconstrained data in wireless sensor networks," in ACS/IEEE International Conference on Computer Systems and Applications, 2005, pp. 904-909.

[7] Y. Yu, B. Krishnamachari, and V. Prasanna, "Data Gathering with Tunable Compression in Sensor Networks," IEEE Transaction on Parallel and Distributed Systems, vol. 19, no. 2, pp. 276-287, 2008.

[8] M. Tahir and R. Farrell, "Optimal Communication-Computation Tradeoff for Wireless Multimedia Sensor Network Lifetime Maximization," in Proc. IEEE Wireless Communications and Networking Conference, 2009, pp. 1-6.

[9] D. Lee and S. Dey, "Adaptive and energy efficient wavelet image compression for mobile multimedia data services," in Proc. IEEE International Conference on Communications, 2002, pp. 2484-2490.

[10] S. Ross, Introduction to Probability Models. Academic Press: San Diego, 2000.

[11] S. Boyd and L. Vandenberghe, Convex Optimization. Cambridge University Press, 2004.

[12] N. Shor, K. Kiwiel, and A. Ruszcaynski, Minimization Methods for Nondifferentiable Functions. Springer-Verlag, New York, 1985. 